\title{
Clinical Study The Obesity Paradox and Cardiorespiratory Fitness
}

\author{
Paul A. McAuley, ${ }^{1}$ Nancy S. Smith, ${ }^{2}$ Brian T. Emerson, ${ }^{2}$ and Jonathan N. Myers ${ }^{3}$ \\ ${ }^{1}$ Department of Human Performance and Sport Sciences, Winston-Salem State University, C024 Anderson, \\ 601 S Martin Luther King Jr Drive, Winston-Salem, NC 27110, USA \\ ${ }^{2}$ Department of Physical Therapy, Winston-Salem State University, Winston-Salem, NC 27110, USA \\ ${ }^{3}$ Department of Cardiology, Division of Cardiovascular Medicine, Veterans Affairs Palo Alto Health Care System, Stanford University, \\ Palo Alto, CA 94304, USA
}

Correspondence should be addressed to Paul A. McAuley, mcauleypa@wssu.edu

Received 25 October 2011; Accepted 8 December 2011

Academic Editor: Carl J. Lavie

Copyright ( 2012 Paul A. McAuley et al. This is an open access article distributed under the Creative Commons Attribution License, which permits unrestricted use, distribution, and reproduction in any medium, provided the original work is properly cited.

\begin{abstract}
Cardiorespiratory fitness as an explanation for the obesity paradox warrants further examination. We evaluated independent and joint associations of cardiorespiratory fitness and adiposity with all-cause mortality in 811 middle-aged (age, $53.3 \pm 7.2$ years) male never smokers without documented cardiopulmonary disease or diabetes from the Veterans Exercise Testing Study (VETS). Cardiorespiratory fitness was quantified in metabolic equivalents (METs) using final treadmill speed and grade achieved on a maximal exercise test. Subjects were grouped for analysis by METs: unfit (lowest third) and fit (upper two-thirds); and by body mass index $\left(\mathrm{kg} / \mathrm{m}^{2}\right)$ : nonobese $(18.5-29.9)$ and obese $(\geq 30.0)$. Associations of baseline fitness and adiposity measures with all-cause mortality were determined by Cox proportional hazards analysis adjusted for age, ethnicity, hypertension, hypercholesterolemia, family history of coronary artery disease, and cardiovascular medication use. In multivariate analysis, mortality risk for obese/fit men did not differ significantly from the nonobese/fit reference group. However, compared to the reference group, nonobese and obese unfit men were $2.2(P=0.01)$ and $1.9(P=0.03)$ times more likely to die, respectively. Cardiorespiratory fitness altered the obesity paradox such that mortality risk was lower for both obese and nonobese men who were fit.
\end{abstract}

\section{Introduction}

In 2002, Gruberg and colleagues [1] coined the term "obesity paradox" to describe their counterintuitive finding that overweight and obese patients with coronary artery disease (CAD) had better outcomes than their normal-weight counterparts. Over the past decade, this unexpected finding has been observed in a range of cardiovascular disease (CVD) pathologies and in several patient groups without CVD [2], suggesting that the obesity paradox is less population specific than originally thought. For example, one recent report [3] found an obesity paradox in patients without CAD as determined by stress single photon emission computed tomography myocardial perfusion imaging. Additionally, two recent studies in patients with $\mathrm{CAD}[4,5]$ found that cardiorespiratory fitness significantly alters the obesity paradox. However, the results of these studies may have been influenced by the presence of smoking and/or other health problems in the normal-weight reference groups.
In an attempt to better isolate the influence of fitness on the obesity paradox, we furthered our study of prospective data from our previous report in middle-aged men with known or suspected CAD who were referred for exercise testing as a part of an extensive medical workup [4]. Within the current study, a healthier cohort of individuals who participated in the Veterans Exercise Testing Study (VETS) were selected for further investigation in an attempt to determine what factors were contributory to mortality. The cohort selected included individuals who had never smoked, had no known baseline cardiopulmonary disease or diabetes, and had a normal exercise test. To avoid the confounding influence of age [6], we confined our investigation to men aged 40 to 65 years. Using 9-year follow-up data, we further explored the obesity paradox in middle-aged men with suspected CAD and assessed the extent to which cardiorespiratory fitness modifies the relation of adiposity to mortality in this population. 


\section{Materials and Methods}

2.1. Study Population. The primary Veterans Exercise Testing Study (VETS) is a prospective epidemiologic investigation of veteran patients begun in 1987. All subjects were referred to exercise testing for either a routine evaluation or to evaluate for exercise-induced ischemia. Participants in the present study were drawn from a cohort of 7775 male veterans referred to one of two university-affiliated Veterans affairs medical centers (Long Beach, Ca, from 1987 to 1991; Palo Alto, Ca, from 1992 to 2003) with followup on all-cause mortality for at least 1 year. All subjects gave informed written consent for participation in the study and the institutional review boards at both sites approved the study. Additional information on study methods and subject characteristics of this cohort has been published elsewhere [7]. For this analysis, we excluded participants: (1) with missing data $(n=175)$; (2) with BMI $<18.5 \mathrm{~kg} / \mathrm{m}^{2}$ $(n=51)$; (3) under 40 and over 65 years of age $(n=$ 2528); (4) with documented CVD (defined as history of myocardial infarction, CAD documented via angiogram, abnormal exercise testing via a graded exercise test, coronary angioplasty, coronary bypass surgery, chronic heart failure, stroke, and/or peripheral vascular disease $)(n=2510)$; (5) with diabetes $(n=321)$; (6) who ever smoked $(n=1379)$. The current analysis included 811 participants (Figure 1).

2.2. Clinical Evaluation and Exercise Testing. A standardized medical examination by a physician, including personal and family histories, was completed for all participants prior to exercise testing. All demographic, clinical, and medication information was obtained from patients' computerized medical records just before the maximal exercise test. Each individual also was asked to verify the computerized information with regard to history of chronic disease, current medications, and cigarette smoking habits. Medications were not changed or stopped before the exercise test occurred.

Maximal exercise testing was performed using an individualized ramp protocol [8] on either a treadmill $(n=$ 764) or an electromagnetically-braked cycle ergometer $(n=$ 47). Before exercise testing, patients completed a Veterans Specific Activity Questionnaire (VSAQ) to estimate their exercise capacity, which allowed most patients to reach maximal exertion within the recommended range of 8 to 12 minutes [9]. In addition to completing the VSAQ, height and weight were measured immediately prior to the exercise test using standard procedures. From this data, body mass index (BMI) was calculated for each individual as weight in kilograms divided by the square of height in meters. Subjects were assigned to one of two categories according to BMI-defined obesity status: nonobese $\left(18.5-29.9 \mathrm{~kg} / \mathrm{m}^{2}\right)$ and obese $\left(\geq 30.0 \mathrm{~kg} / \mathrm{m}^{2}\right)$.

Prior to exercise testing, supine resting heart rate and blood pressure were assessed after 5 minutes. Blood pressure was recorded on alternate minutes throughout the test, and a 12-lead electrocardiogram was continuously monitored and printed every minute. A computerized system automatically

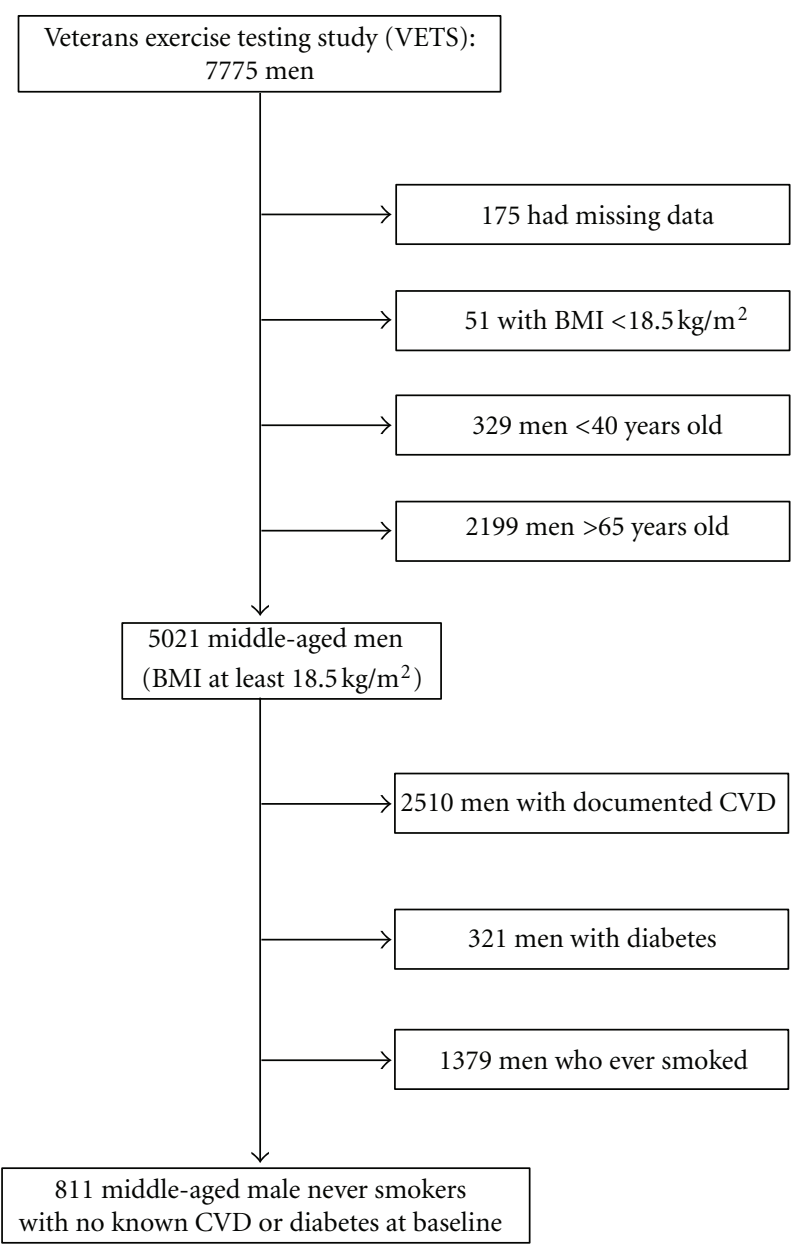

FIgURE 1: Flowchart of participant selection.

increased workload after an individualized walking speed (treadmill) or watts (cycle) was established and predicted values for maximal exercise capacity were entered. Subjects were encouraged to exercise until volitional fatigue in the absence of symptoms or other indicators of ischemia. The patient's subjective level of exertion was assessed by the Borg 6-20 scale [10]. Standard clinical criteria for terminating the tests (e.g., fall in systolic blood pressure, ST-segment depression $>2.0 \mathrm{~mm}$, dangerous arrhythmias) were followed [11], but no heart rate or time limit was imposed and a maximal effort was encouraged.

Cardiorespiratory fitness was calculated from final treadmill speed and grade or cycle ergometer watts as metabolic equivalents (METs) using standard American College of Sports Medicine equations (one MET is defined as the energy expended at rest, which is equivalent to an oxygen consumption of $3.5 \mathrm{~mL} \cdot \mathrm{kg}^{-1} \cdot \mathrm{min}^{-1}$ ) [11]. A standardized method for categorizing fitness does not exist. However, in epidemiological studies, the lowest third for the population is frequently defined as "low fitness" [12]. Therefore, we grouped participants for analysis as "unfit" and "fit" according to the lower third and upper two-thirds of cardiorespiratory fitness for the population, respectively. 
TABLE 1: Baseline characteristics of the study population.

\begin{tabular}{|c|c|c|c|}
\hline Characteristic & BMI, 18.5-29.9 $(n=511)$ & BMI, $\geq 30.0(n=300)$ & $P$-value \\
\hline Age, years & $53.8 \pm 7.5$ & $52.5 \pm 6.7$ & 0.01 \\
\hline Non-Hispanic white, \% & 73.4 & 66.3 & 0.03 \\
\hline Cardiorespiratory fitness, METs* & $11.2 \pm 3.5$ & $9.2 \pm 3.0$ & $<0.001$ \\
\hline Unfit, \%* & 25.2 & 47.0 & $<0.001$ \\
\hline Fit, \%* & 74.8 & 53.0 & \\
\hline Resting heart rate (bpm) & $76.1 \pm 14.1$ & $79.2 \pm 14.7$ & 0.003 \\
\hline \multicolumn{4}{|l|}{ Resting blood pressure (mmHg) } \\
\hline Systolic & $130.3 \pm 18.2$ & $134.9 \pm 18.7$ & $<0.001$ \\
\hline Diastolic & $83.9 \pm 10.6$ & $86.4 \pm 12.1$ & 0.002 \\
\hline Hypertension, \% & 32.3 & 56.3 & $<0.001$ \\
\hline Hypercholesterolemia, \% & 33.6 & 44.9 & 0.002 \\
\hline \multicolumn{4}{|l|}{ Cardiovascular medication use, $\%$} \\
\hline Betablockers & 9.8 & 20.0 & $<0.001$ \\
\hline Calcium antagonists & 10.0 & 19.0 & $<0.001$ \\
\hline Antihypertensives & 16.6 & 23.3 & 0.02 \\
\hline Antiarrhythmics & 1.6 & 1.3 & 0.79 \\
\hline ACE inhibitors & 4.7 & 12.7 & $<0.001$ \\
\hline Anticoagulants & 9.4 & 13.7 & 0.06 \\
\hline Statins & 4.3 & 4.0 & 0.83 \\
\hline Diuretics & 1.0 & 2.7 & 0.06 \\
\hline All-cause deaths, $\%$ & 11.9 & 11.3 & 0.80 \\
\hline Followup, years & $8.9 \pm 5.4$ & $8.3 \pm 5.0$ & 0.12 \\
\hline
\end{tabular}

Data shown are mean \pm SD unless otherwise specified. $P$ values were calculated from the $t$-test for continuous variables and chi-square for categorical variables. BMI, body mass index; METs, metabolic equivalents.

$* 1 \mathrm{MET}=3.5 \mathrm{~mL} / \mathrm{kg} / \mathrm{min}$ oxygen uptake, calculated from final treadmill speed and grade during maximal exercise test; lower third $(<9 \mathrm{METs})$ and upper two-thirds ( $\geq 9 \mathrm{METs})$ of cohort were classified as unfit and fit, respectively.

2.3. Mortality Surveillance. Participants were followed for at least 1 year from their baseline examination until their death or until 30 December, 2004. We recorded death dates from the Veterans Affairs Beneficiary Identification and Record Locator System File. The Social Security Death Index was used to match all subjects to their record according to Social Security number. Accuracy of deaths was reviewed by two clinicians blinded to exercise test results and confirmed using the Veterans Affairs computerized medical records.

2.4. Statistical Analysis. Number Crunching Statistical Software (Kaysville, UT) was used for all statistical analyses. The mean and SD of each variable were calculated with participants categorized as nonobese and obese. Cox proportional hazards analyses were used to assess separate and combined associations of cardiorespiratory fitness and adiposity at baseline with the risk of all-cause mortality. To test for the interactions of cardiorespiratory fitness, adiposity, and mortality, we calculated an interaction term for METs and BMI as continuous variables entered into an ageadjusted model. For stratified analyses, we selected nonobese and fit men as the reference group and calculated hazard ratios (HRs) of all-cause mortality for the remaining three fitness-obesity status groups (i.e., nonobese/unfit, obese/fit, and obese/unfit). Covariates included age (years), ethnicity
(non-Hispanic White, African-American, Hispanic, AsianPacific Islander, or unknown), hypertension (yes or no), hypercholesterolemia (yes or no), family history of coronary artery disease (yes or no), and CVD medication use (yes or no for each medication listed in Table 1). We used two tailed $t$-tests to compare means for continuous variables and Chisquare comparisons for categorical variables and regarded a $P$ value of $<0.05$ as statistically significant.

\section{Results}

After a mean followup of 8.7 years (range, 1.2 to 17.6 years), 95 patients died, for an annual mortality rate of $1.3 \%$. Obese men, who represented $37 \%$ of the cohort, were comparable to nonobese men in age and ethnic composition, but were nearly twice as likely to be classified as unfit, had a higher prevalence of hypertension (56\% versus $32 \%)$ and hypercholesterolemia (45\% versus 34\%) and a higher use of four cardiovascular medications: beta-blockers (20\% versus $10 \%)$, calcium antagonists (19\% versus $10 \%)$, antihypertensives (23\% versus $17 \%$ ), and ACE inhibitors ( $13 \%$ versus $5 \%$ ) (Table 1$)$. Neither mean follow-up duration nor crude mortality rate differed significantly by obesity status. 
TABLE 2: Independent associations of baseline fitness and BMI with all-cause mortality.

\begin{tabular}{|c|c|c|c|c|c|c|}
\hline \multirow{2}{*}{ Variables } & \multirow{2}{*}{$n$} & \multirow{2}{*}{ Deaths (\%) } & \multicolumn{2}{|c|}{ Model $1^{*}$} & \multicolumn{2}{|c|}{ Model $2^{\dagger}$} \\
\hline & & & $\operatorname{HR}(95 \% \mathrm{CI})$ & $P$ Value & $\operatorname{HR}(95 \% \mathrm{CI})$ & $P$ Value \\
\hline \multicolumn{7}{|c|}{ As continuous variables } \\
\hline Per MET & 811 & $95(11.7)$ & $0.91(0.85-0.97)$ & 0.002 & $0.90(0.84-0.96)$ & 0.001 \\
\hline Per kg/m² & 811 & $95(11.7)$ & $0.99(0.96-1.09)$ & 0.91 & $0.98(0.93-1.02)$ & 0.25 \\
\hline \multicolumn{7}{|c|}{ As categorical variables } \\
\hline Fit & 541 & $45(8.3)$ & 1 (reference) & & 1 (reference) & \\
\hline Unfit & 270 & $50(18.5)$ & $2.05(1.34-3.15)$ & 0.001 & $2.26(1.43-3.56)$ & $<0.001$ \\
\hline Nonobese & 511 & $61(11.9)$ & 1 (reference) & & 1 (reference) & \\
\hline Obese & 300 & $34(11.3)$ & $1.13(0.72-1.76)$ & 0.59 & $0.92(0.58-1.46)$ & 0.72 \\
\hline
\end{tabular}

CI, confidence interval; HR, hazard ratio.

* Adjusted for age, ethnicity, hypertension, hypercholesterolemia, family history of heart disease, and cardiovascular medication use.

${ }^{\dagger}$ Adjusted for above plus METs (for BMI) and BMI (for METs) as continuous variables.

Multivariate proportional HRs (95\% confidence intervals [CI]) for independent associations of cardiorespiratory fitness and adiposity measures with all-cause mortality are shown in Table 2. In the fully adjusted model, HRs (95\% CI) were $0.90(0.84-0.96 ; P=0.001)$ per MET increment and $0.98(0.93-1.02 ; P=0.25)$ per unit BMI $\left(\mathrm{kg} / \mathrm{m}^{2}\right)$ increment. The results for the categorical variables are also shown in Table 2. Whereas unfit men $(n=270)$ were 2.3 times more likely to die $(P<0.001)$ compared to the reference group of 541 fit men, obese men $(n=300)$ were no more likely to die $(P=0.72)$ compared to the reference group of 511 nonobese men.

Testing of the interaction model revealed a significant interaction between BMI and METs $(P<0.001)$, thus we proceeded with the stratified analysis (Figure 2). Unadjusted HRs (95\% CIs) from Kaplan-Meier analysis for nonobese/unfit, obese/fit, and obese/unfit were 2.99 (1.635.47), 1.03 (0.53-2.00), and 2.18 (1.17-4.08). Using the group of 382 nonobese/fit men as the reference, multivariate HRs (95\% CI) were 2.19 (1.29-3.73) for 129 nonobese/unfit men and 1.86 (1.07-3.23) for 141 obese/unfit men. However, all-cause mortality risk for 159 obese/fit men did not differ significantly from the reference group $(P=0.85)$.

\section{Discussion}

Overall, the results demonstrate that obese men were at no greater risk for 9 year all-cause mortality than nonobese men. This is the first study to confirm the presence of an obesity paradox in a population of healthier middle-aged men in the VETS cohort and extends previous observations of an obesity paradox in healthy older men in the VETS [13]. Though it is unlikely that a single variable could fully explain the obesity paradox phenomenon, we nevertheless demonstrated that cardiorespiratory fitness level altered the obesity paradox: unfit men were roughly twice as likely to die as fit men regardless of obesity status, and fit men who were obese survived as well as nonobese/fit men.

Some have suggested that the obesity paradox is due to poorer health [14] or the negative influence of smoking [15] in the reference group of normal-weight subjects.

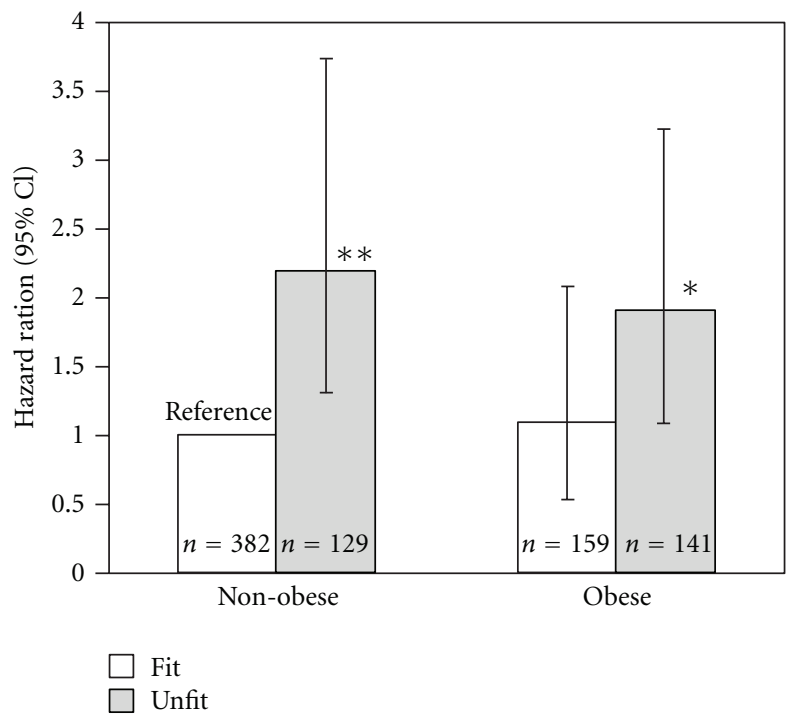

FIGURE 2: Multivariate hazard ratios for all-cause mortality by obesity status and fitness level. Each bar represents the relative risk after adjustment for age, ethnicity, hypertension, hypercholesterolemia, family history of coronary artery disease, and cardiovascular medication use with the relative risk of nonobese (BMI 18.5$29.9 \mathrm{~kg} / \mathrm{m}^{2}$ ) and fit (>9 METs) set at 1.0. Grey bars represent unfit (lowest third of distribution) and dark bars fit (upper two-thirds of distribution). Error bars represent 95\% confidence intervals. Differed from reference: ${ }^{*} P<0.05$; ${ }^{* *} P<0.01$.

In this study, we attempted to avoid these problems by selecting a group of middle-aged men referred to exercise testing for clinical reasons but deemed apparently healthy on the basis of a normal exercise test, no prior history of cardiopulmonary disease or diabetes, and who never smoked. Furthermore, by including exercise test data in the analysis, we attempted to isolate the independent influence of cardiorespiratory fitness on the obesity paradox phenomenon.

Several theories have been advanced in an attempt to explain the obesity paradox, including the finding that in most studies the obese population is younger [16] and the observed protective effect may be limited to the relatively 
short follow-up period of most studies [17]. While these factors may explain the obesity paradox in many prospective studies, the subjects in our study were homogeneous with respect to age and were followed for mortality for nearly 9 years. Others have suggested that obese patients may receive better medical care sooner [18], a possibility that cannot be ruled out in the present study.

Another line of criticism regarding the obesity paradox focuses on the limitations of BMI as a measure of adiposity, arguing that higher BMI may simply mean greater muscle mass which in turn implies a more favorable health status [19]. However, Pearson correlations in the range of 0.7 to 0.8 between BMI and percentage body fat have been reported for both men and women $[20,21]$. And a recent systematic review and meta-analysis of the diagnostic performance of BMI to identify obesity defined by body adiposity found that when BMI is $\geq 30 \mathrm{~kg} / \mathrm{m}^{2}$, it is an excellent predictor of excess adiposity in both sexes [22]. The issue of BMI versus percentage body fat in obesity paradox was addressed by Lavie and colleagues [23] in a study of 209 heart failure patients, and in their more recent study of 529 patients with CAD [24]. Both studies found that both higher BMI and higher percentage body fat were independent predictors of better survival. And a recent study of 13,155 men with hypertension from the Aerobics Center Longitudinal Study (ACLS), found that CVD mortality risk was similar for BMI and percentage body fat [25]. Therefore, BMI appears to be an adequate marker of overall adiposity in population studies.

Few studies of obesity paradox include information on cardiorespiratory fitness $[4,5,26]$, which greatly modifies mortality risk. For example, in our previous VETS report that included middle-aged male veterans with known or suspected CAD, an obesity paradox was present in the lowfitness group, but absent among participants registering high fitness. We did not observe such a dichotomy in the present study and the reason for this is likely due to the better health status of the reference group. However, veterans are a select group in that they have to meet military enlistment standards that include minimum fitness and maximum body weight criteria. Therefore, the results of the present study may be influenced by a "veteran effect" (i.e., all participants were previously fit and nonobese at the time of their enlistment).

Our study has several limitations: (1) since BMI was the only anthropometric measurement obtained, we were not able to evaluate body fat distribution characteristics; (2) all participants in this study were men who had prior military service and were referred to exercise testing for clinical reasons. Therefore, our results may not be generalized to civilians or women; (3) though our selection procedures enabled us to deem these subjects healthy, we cannot rule out the possibility that undetected CVD or other illnesses were present; (4) the extent of fitness improvement in adults, or the influence this may have on mortality, cannot be determined from the present investigation; (5) we did not have sufficient information about diet or physical activity patterns to study these factors; (6) since we only have baseline data on weight, exercise capacity, and other exposures, we do not know if changes in any of these variables occurred during followup and how this might have influenced the results; (7) data on peak $\mathrm{VO}_{2}$ were not available, which is the most accurate measure of cardiorespiratory fitness.

In conclusion, while our study suggests that the obesity paradox extends to healthier populations with only suspected $\mathrm{CAD}$, cardiorespiratory fitness level rather than obesity accounted for differences in mortality risk. Whereas low fitness more than doubled all-cause mortality risk in this population, obesity provided neither protection against nor contributed to mortality. Thus, fitness predicted mortality independent of BMI. Our findings suggest that strategies to reduce mortality risk among obese adults should emphasize preserving or increasing cardiorespiratory fitness. Future studies should obtain simultaneous measures of fitness and adiposity and how changes in these parameters affect mortality and other health outcomes.

\section{Conflict of Interests}

The authors have no conflict of interests to report.

\section{References}

[1] L. Gruberg, N. J. Weissman, R. Waksman et al., "The impact of obesity on the short-term and long-term outcomes after percutaneous coronary intervention: the obesity paradox?" Journal of the American College of Cardiology, vol. 39, no. 4, pp. 578-584, 2002.

[2] P. A. McAuley and S. N. Blair, "Obesity paradoxes," Journal of Sports Sciences, vol. 29, no. 8, pp. 773-782, 2011.

[3] S. Uretsky, A. Supariwala, P. Singh et al., "Impact of weight on long-term survival among patients without known coronary artery disease and a normal stress SPECT MPI," Journal of Nuclear Cardiology, vol. 17, no. 3, pp. 390-397, 2011.

[4] P. A. McAuley, P. F. Kokkinos, R. B. Oliveira, B. T. Emerson, and J. N. Myers, "Obesity paradox and cardiorespiratory fitness in 12, 417 male veterans aged 40 to 70 years," Mayo Clinic Proceedings, vol. 85, no. 2, pp. 115-121, 2010.

[5] K. Goel, R. J. Thomas, R. W. Squires et al., "Combined effect of cardiorespiratory fitness and adiposity on mortality in patients with coronary artery disease," American Heart Journal, vol. 161, no. 3, pp. 590-597, 2011.

[6] R. Bender, K. H. Jöckel, C. Trautner, M. Spraul, and M. Berger, "Effect of age on excess mortality in obesity," Journal of the American Medical Association, vol. 281, no. 16, pp. 1498-1504, 1999.

[7] P. McAuley, J. Myers, B. Emerson et al., "Cardiorespiratory fitness and mortality in diabetic men with and without cardiovascular disease," Diabetes Research and Clinical Practice, vol. 85, no. 3, pp. e30-e33, 2009.

[8] J. Myers, N. Buchanan, D. Walsh et al., "Comparison of the ramp versus standard exercise protocols," Journal of the American College of Cardiology, vol. 17, no. 6, pp. 1334-1342, 1991.

[9] J. Myers, W. Herbert, P. Ribisl, and V. F. Froelicher, "A nomogram to predict exercise capacity from a specific activity questionnaire and clinical data," American Journal of Cardiology, vol. 73, no. 8, pp. 591-596, 1994.

[10] G. A. Borg, "Psychophysical bases of perceived exertion," Medicine and Science in Sports and Exercise, vol. 14, no. 5, pp. 377-381, 1982. 
[11] American College of Sports Medicine, Guidelines for Exercise Testing and Prescription, Williams \& Wilkins, Baltimore, Md, USA, 8th edition, 2009.

[12] P. A. McAuley and S. N. Blair, The Metabolic Syndrome, WileyBlackwell, Oxford, UK, 2nd edition, 2011.

[13] P. McAuley, J. Pittsley, J. Myers, J. Abella, and V. F. Froelicher, "Fitness and fatness as mortality predictors in healthy older men: the veterans exercise testing study," Journals of Gerontology A, vol. 64, no. 6, pp. 695-699, 2009.

[14] A. Habbu, N. M. Lakkis, and H. Dokainish, "The obesity paradox: fact or fiction?" American Journal of Cardiology, vol. 98, no. 7, pp. 944-948, 2006.

[15] C. J. Lavie, H. O. Ventura, and R. V. Milani, "The "obesity paradox": is smoking/lung disease the explanation?" Chest, vol. 134, no. 5, pp. 896-898, 2008.

[16] A. H. Wu, B. Pitt, S. D. Anker, J. Vincent, M. Mujib, and A. Ahmed, "Association of obesity and survival in systolic heart failure after acute myocardial infarction: potential confounding by age," European Journal of Heart Failure, vol. 12, no. 6, pp. 566-573, 2010.

[17] A. Nigam, R. S. Wright, T. G. Allison et al., "Excess weight at time of presentation of myocardial infarction is associated with lower initial mortality risks but higher long-term risks including recurrent re-infarction and cardiac death," International Journal of Cardiology, vol. 110, no. 2, pp. 153-159, 2006.

[18] T. Lancefield, D. J. Clark, N. Andrianopoulos et al., "Is there an obesity paradox after percutaneous coronary intervention in the contemporary era? An analysis from a multicenter Australian registry," Journal of the American College of Cardiology, vol. 3, no. 6, pp. 660-668, 2010.

[19] C. Kragelund and T. Omland, "A farewell to body-mass index?” The Lancet, vol. 366, no. 9497, pp. 1589-1591, 2005.

[20] P. J. Schreiner, J. G. Terry, G. W. Evans, W. H. Hinson, J. R. Crouse Jr., and G. Heiss, "Sex-specific associations of magnetic resonance imaging-derived intra- abdominal and subcutaneous fat areas with conventional anthropometric indices: the atherosclerosis risk in communities study," American Journal of Epidemiology, vol. 144, no. 4, pp. 335-345, 1996.

[21] S. W. Farrell, S. J. Fitzgerald, P. A. McAuley, and C. E. Barlow, "Cardiorespiratory fitness, adiposity, and all-cause mortality in women," Medicine and Science in Sports and Exercise, vol. 42, no. 11, pp. 2006-2012, 2010.

[22] D. O. Okorodudu, M. F. Jumean, V. M. Montori et al., "Diagnostic performance of body mass index to identify obesity as defined by body adiposity: a systematic review and meta-analysis," International Journal of Obesity, vol. 34, no. 5, pp. 791-799, 2010.

[23] C. J. Lavie, A. F. Osman, R. V. Milani, and M. R. Mehra, "Body composition and prognosis in chronic systolic heart failure: the obesity paradox," American Journal of Cardiology, vol. 91, no. 7, pp. 891-894, 2003.

[24] C. J. Lavie, R. V. Milani, S. M. Artham, D. A. Patel, and H. O. Ventura, "The obesity paradox, weight loss, and coronary disease," American Journal of Medicine, vol. 122, no. 12, pp. 1106-1114, 2009.

[25] P. A. McAuley, X. Sui, T. S. Church, J. W. Hardin, J. N. Myers, and S. N. Blair, "The joint effects of cardiorespiratory fitness and adiposity on mortality risk in men with hypertension," American Journal of Hypertension, vol. 22, no. 10, pp. 10621069, 2009.

[26] N. P. Johnson, E. Wu, R. O. Bonow, and T. A. Holly, "Relation of exercise capacity and body mass index to mortality in patients with intermediate to high risk of coronary artery disease," American Journal of Cardiology, vol. 102, no. 8, pp. 1028-1033, 2008. 


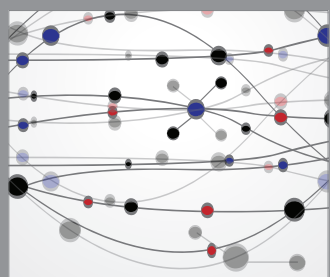

The Scientific World Journal
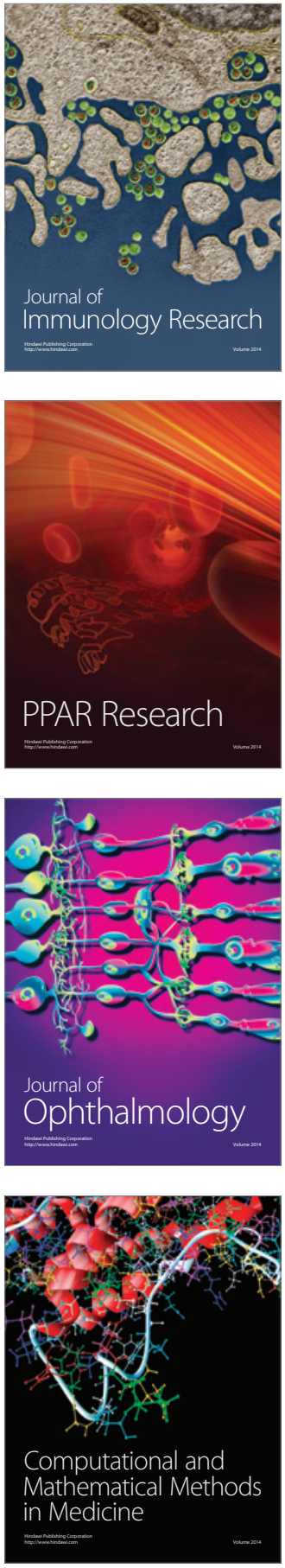

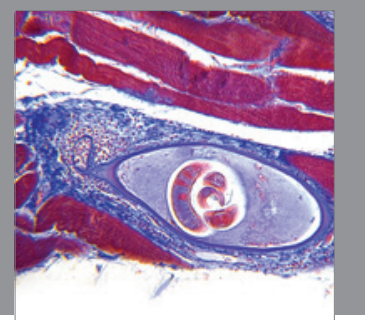

Gastroenterology

Research and Practice
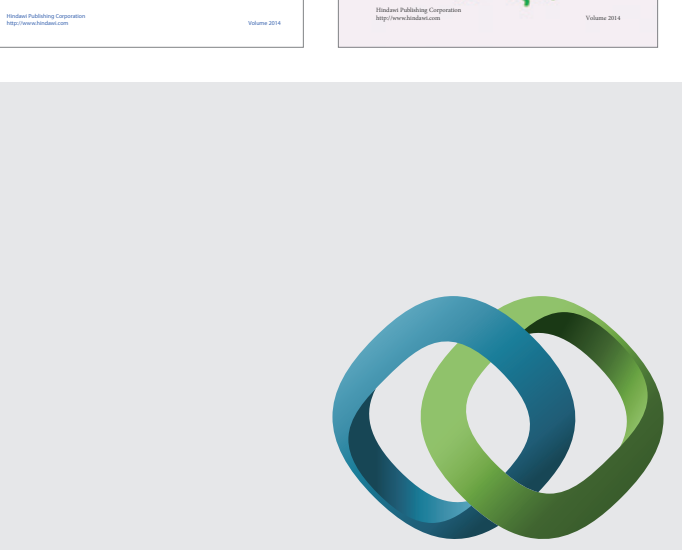

\section{Hindawi}

Submit your manuscripts at

http://www.hindawi.com
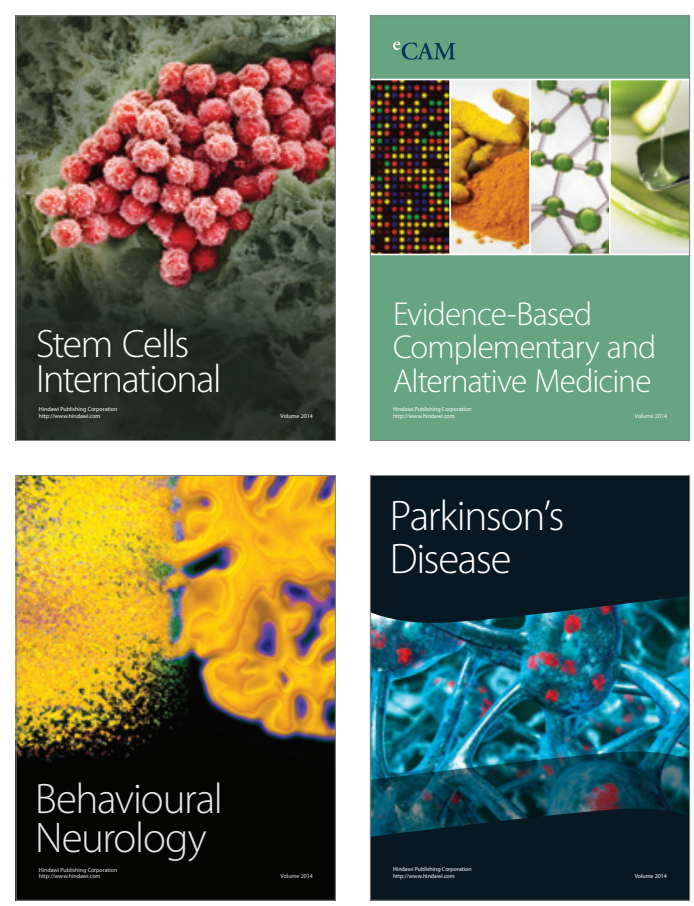

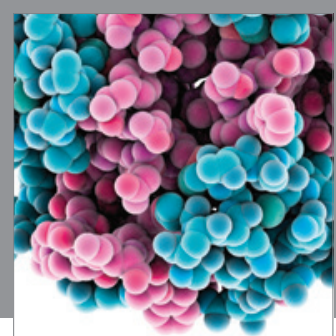

Journal of
Diabetes Research

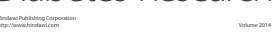

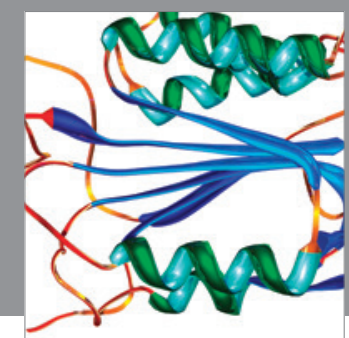

Disease Markers
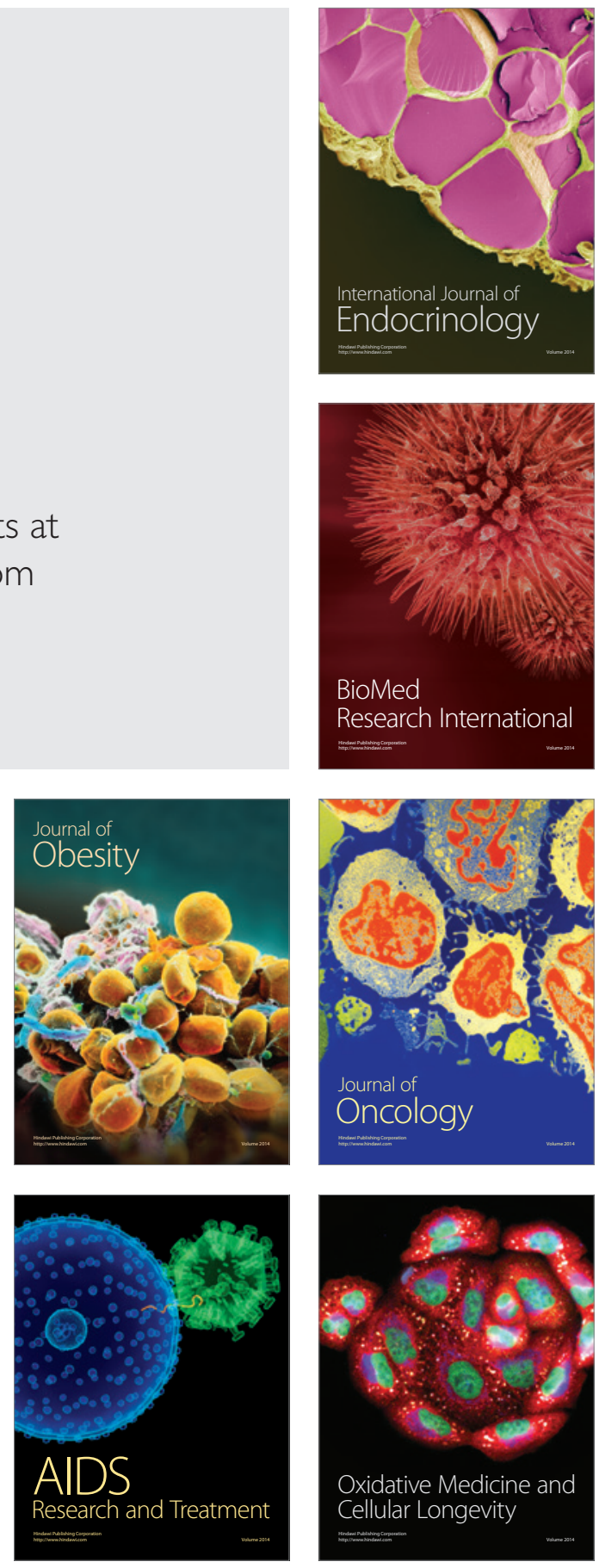